\title{
The detection of cognitive state transitions by stability changes in event-related cortical field potentials
}

\author{
Hualou Liang*, Mingzhou Ding, Steven L. Bressler \\ Center for Complex Systems and Brain Sciences, Florida Atlantic University, P.O. Box 3091, \\ 777 Glades Road, Boca Raton, FL 33431, USA
}

\begin{abstract}
Cognitive tasks are characterized by sequences of stable states, which are thought to reflect distinct steps of neurocognitive processing. Here, we investigate a stability measure, derived from adaptive multivariate autoregressive (AMVAR) modeling of cortical field potentials, as an index for relating changes in large-scale neural activity to changes in cognitive state. We show that this stability measure can be used to decide the optimal window length for AMVAR modeling and to detect state transitions related to external sensory or motor events. By using this approach, we demonstrate clear differentiation of GO and NO-GO processes in a macaque monkey performing a visuomotor pattern discrimination task. Moreover, we are able to identify regional differences in state transitions, apparently reflecting regional information processing differences. (C) 2001 Elsevier Science B.V. All rights reserved.
\end{abstract}

Keywords: Local field potentials; State dynamics; Cerebral cortex; Stability

\section{Introduction}

Cognitive tasks are characterized by sequences of stable states, which are thought to reflect distinct steps of neurocognitive processing. An important question in cognitive neuroscience is how to detect these states and their transitions by analysis of neural activity. This problem involves ascertaining to what extent these internal states correspond to external sensory or motor events, and to what degree different brain regions contribute to the process.

\footnotetext{
* Corresponding author. Tel.: + 1-561-297-0108; fax: + 1-561-297-3634.

E-mail address: liang@walt.ccs.fau.edu (H. Liang).
} 
The rapid state change of cognitive processing gives rise to nonstationary neural activity. The adaptive multivariate autoregressive (AMVAR) [2] modeling approach deals with this nonstationarity by segmenting the series into approximately stationary time series windows and modeling each segment as a stationary process. This naturally raises the question of how to achieve the proper window size in order to trace the rapid dynamic changes inherent in neurophysiological data. To answer this question, we devised a measure, called the stability index, to gauge stationarity and allow us to choose appropriate window sizes. The most intriguing aspect of this measure is that it may be used to explore the relationship between changes in field potential stationarity and the occurrence of external sensory or motor events, namely a visual stimulus and the execution of a GO or NO-GO response. By using this approach, we demonstrate clear differentiation of GO and NO-GO processes in a macaque monkey performing a visuomotor pattern discrimination task. Moreover, we are able to identify regional differences in state transitions, apparently reflecting regional information processing differences. Thus AMVAR modeling may offer a new tool to reveal different information processing modes that depend on behavioral contingencies.

\section{Methods}

\subsection{Experimental methods [1]}

Surface-to-depth event-related local field potential (LFP) data were recorded from highly trained macaque monkeys performing a visuomotor task in which they discriminated dot patterns arranged as either diamonds or lines; each pattern could be either right- or left-slanted. In each recording session, a GO response was contingent on one pattern type and a NO-GO response on the other. The contingency was reversed across sessions. Trials from sessions having mixed response contingencies were pooled, forming balanced data sets that differed only in stimulus pattern (diamond vs. line) or response type (GO vs. NO-GO).

LFPs were simultaneously recorded from bipolar electrodes chronically implanted at 15 sites distributed in striate, prestriate, parietal, inferior temporal, motor and frontal areas of the cerebral hemisphere contralateral to the responding hand (Fig. 1). The LFPs were sampled at $200 \mathrm{~Hz}$ from around $115 \mathrm{~ms}$ prior to stimulus onset to $500 \mathrm{~ms}$ after stimulus onset in each trial of a session.

\subsection{The stability index}

Let $\mathbf{X}_{t}=\left[x_{1 t}, x_{2 t}, \ldots, x_{p t}\right]^{\mathrm{T}}$ be $p$ channels of LFPs. The multivariate autoregressive (MVAR) model is given by

$$
\sum_{k=0}^{m} \mathbf{A}_{k} \mathbf{X}_{t-k}=\mathbf{E}_{t}
$$

where $\mathbf{E}_{t}$ is uncorrelated noise with covariance matrix $\boldsymbol{\Sigma}$, and $\mathbf{A}_{k}$ are $p \times p$ coefficient matrices $\left(A_{0}=I\right.$, identity matrix) which can be obtained by solving the multivariate 


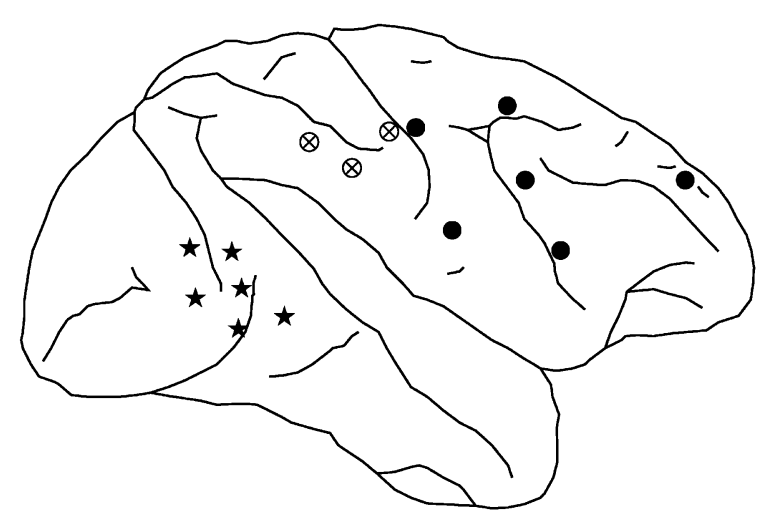

Fig. 1. Map of the right hemisphere of monkey GE, showing the 15 electrode sites. Sites with different symbols represent ventral $(\star)$, parietal $(\otimes)$ and frontal $(\bigcirc)$ regions, a division used in this paper for identifying regional differences in state transitions.

Yule-Walker Equations (of size $m p^{2}$ ) using the Levinson, Wiggins and Robinson (LWR) algorithm [4]. The model order $m$ is determined by the Akaike information criterion (AIC) [3].

The characteristic equation of the MVAR model in (1) can be described as

$$
\operatorname{det}\left(\lambda^{m} \mathbf{I}+\lambda^{m-1} \mathbf{A}_{1}+\cdots+\lambda \mathbf{A}_{m-1}+\mathbf{A}_{m}\right)=0 .
$$

where the condition $|\lambda|<1$ is required to ensure the stability of the fitted model. The stability index (SI) is therefore defined as

$$
\mathrm{SI}=\log \left|\lambda_{1}\right|
$$

where $\lambda_{1}$ denotes the largest eigenvalue of characteristic Eq. (2). By this definition, a fitted model is stable only if the SI is less than 0 .

AMVAR analysis was applied in a 50-ms window that was stepped point by point through the task. (The determination of optimal window length is given below.) In each window, data from all trials and all electrodes were used to estimate the MVAR model, and the SI was computed.

\section{Results}

The data set consisted of 888 trials taken from multiple sessions recorded from monkey GE. Data sets balanced for response type (GO vs. NO-GO) and stimulus pattern (diamond vs line) were used in the analysis. The GO vs NO-GO comparison was used for the detection of cognitive state transitions related to the external motor event. The diamond and line data sets were not expected to differ in relation to the motor event since they were balanced for response type. Therefore, the diamond vs line comparison served as control. 
How to choose the proper window size is a crucial question in the AMVAR modeling of time series. Fig. 2 illustrates the procedure for determining the appropriate window length based on the SI. Fig. 2 reveals that: (1) all the fitted models with different window lengths are stable since their SIs are all less than $0 ;(2)$ as the window length increases, the SI becomes smoother, thus smearing the temporal dynamics; (3) to preserve the variability and maintain the smoothness of the SI, the 10-point-long window is a good compromise. More detailed discussion on choosing window size can be found in [2]. Based on this observation, in what follows, all results are based on the 10-point window length.

The stability index revealed several results. (1) There was a clear-cut differentiation of GO and NO-GO conditions only preceding and during the time of the movement in the GO condition (Fig. 3, left). (2) In contrast, no such discrimination was found in the diamond vs. line comparison (Fig. 3, right). (3) In both comparisons, the stability index was not appreciably different during early visual processing. (4) Ventral cortical sites, i.e. striate, prestriate, and inferotemporal sites, were primarily responsible for a peak during stimulus processing, which did not depend on response type or stimulus pattern (Fig. 4, left column). (5) Frontal cortical sites contributed to the differentiation of response conditions precisely at the mean response onset time (Fig. 4, right column). (6) The parietal region (Fig. 4, middle column) appeared to have stability properties that were intermediate between the ventral and frontal regions.

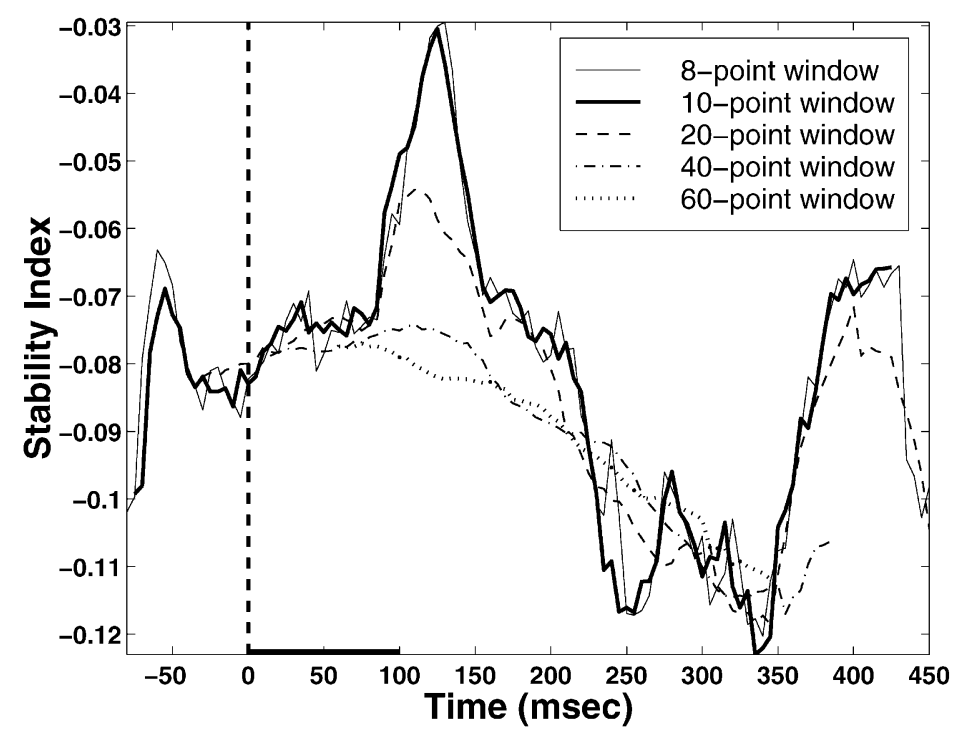

Fig. 2. Stability indexes computed with different window lengths, showing that the 10-point-long window is a good choice for AMVAR modeling. 

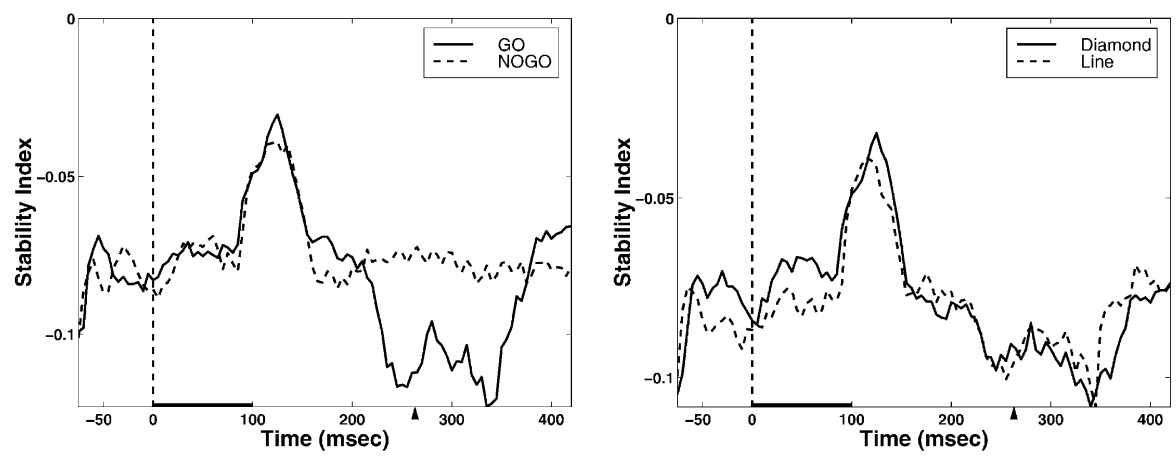

Fig. 3. Stability indexes calculated from all of the 15 channels for GO and NO-GO (left) and for diamond and line (right). Vertical dashed lines indicate the stimulus onset. The stimulus lasts $100 \mathrm{~ms}$ marked by the dark horizontal bar. The upward arrowhead represents the mean response onset time.
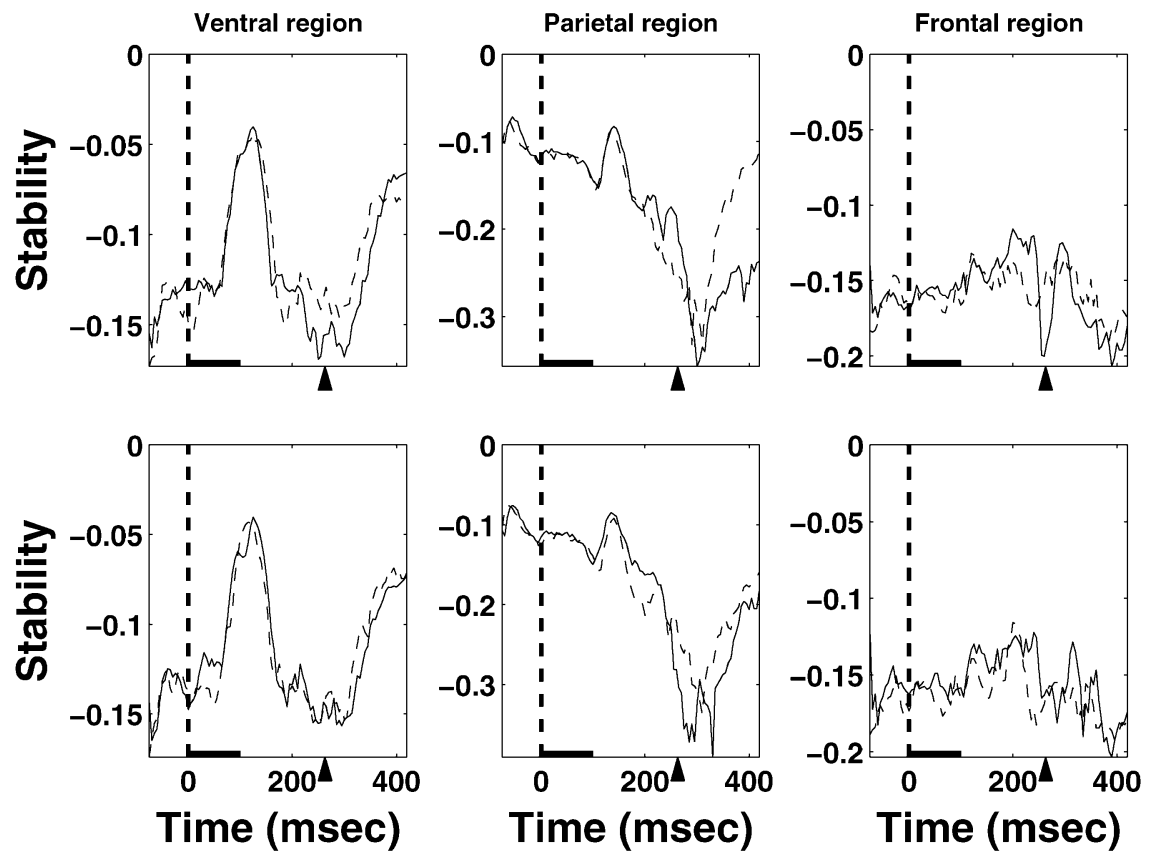

Fig. 4. Top: stability indexes for GO (solid) and NO-GO (dashed) from left to right are derived from channels in ventral, parietal and frontal regions, respectively. Bottom: stability indexes for diamond (solid) and line (dashed) conditions from the same cortical regions as that in the top row are shown as controls. See Fig. 3 for detailed notation. 


\section{Conclusions}

We conclude that the stability index is a useful tool to study state dynamics of neural activity during cognitive task performance. It can be used to distinguish different behavioral modes, e.g. GO and NO-GO, and to identify the dependence of state changes on different cortical areas. The findings presented here, from one monkey, also appear to apply in three other monkeys we have studied.

\section{Acknowledgements}

Supported by the NIMH grant MH-58190, NSF grant IBN-09723240, and ONR grant 99-1-0062.

\section{References}

[1] S.L. Bressler, R. Coppola, R. Nakamura, Episodic multiregional cortical coherence at multiple frequencies during visual task performance, Nature 366 (1993) 153-156.

[2] M. Ding, S.L. Bressler, W. Yang, H. Liang, Short window spectral analysis of cortical event-related potentials by Adaptive multivariate autoregressive (AMVAR) modeling: data preprocessing, model validation, and variability assessment by bootstrapping, Biol. Cybernet 83 (2000) 35-45.

[3] S.L. Marple, Digital Spectral Analysis with Applications, Prentice-Hall, Englewood Cliffs, NJ, 1987.

[4] M. Morf, A. Vieira, D. Lee, T. Kailath, Recursive multichannel maximum entropy spectral estimation, IEEE Trans. Geosci. Electron. 16 (1978) 85-94. 